\title{
CryoEM of Molecular Machines
}

\begin{abstract}
Wah Chiu
National Center for Macromolecular Imaging, Baylor College of Medicine, Houston, TX 77030

USA

wah@bcm.edu

Electron cryo-microscopy (cryoEM) is in the midst of a rapid advance in resolving biological structures of molecular machines previously either difficult or impossible to attain, at near atomic resolutions. In our Center, we have solved cryoEM structures of molecular machines, including viruses, chaperonins, protein complexes and membrane proteins, to the level where full-atom models of the protein components can be obtained from cryoEM maps. Examples will be presented illustrating how novel biochemical knowledge is derived and validated from cryoEM structures.
\end{abstract}

